\title{
Evaluation of $g$ Values for Compounds with a Paramagnetic Ion
}

\author{
B. N. Misra, S. D. Sharma, and S. K. Gupta \\ Department of Physics, University of Allahabad, Allahabad, India \\ (Z. Naturforsch. 28a, 246-248 [1973]; received 9 September 1972)
}

\begin{abstract}
A general and approximate method based on the concept of a point charge point dipole method is applied to some compounds to estimate the $\mathrm{g}$ values of molecules with a paramagnetic ion in the presence of a strong crystalline electric field. The paramagnetic ion consideren is $\mathrm{Cu}^{2+}$ and the compounds are $\mathrm{Cu}(\mathrm{en})_{2} \mathrm{Cl}_{2} \cdot 2 \mathrm{H}_{2} \mathrm{O}, \mathrm{Cu}\left(\mathrm{NH}_{3}\right)_{4} \mathrm{SO}_{4} \cdot \mathrm{H}_{2} \mathrm{O}$ and $\mathrm{Cu}\left(\mathrm{NH}_{4}\right)_{2}$ $\left(\mathrm{SO}_{4}\right)_{2} \cdot 6 \mathrm{H}_{2} \mathrm{O}$. An attempt is made to study the model for nonionic structures. An allowance is made for covalent bonding. The presence of the Jahn-Teller effect is accounted for. The results agree fairly well with experimental values.
\end{abstract}

\section{Introduction}

On the basis of experimental data the constants occuring in the spin-Hamiltonian are calculated and from this exact information about the symmetry of the crystalline field and the nature of the neighbours of the paramagnetic ion can be obtained. One of the constants, the spectroscopic splitting factor $(g)$, is equal to 2 for free spin but, owing to imperfect quenching of the orbital levels, is generally different and varies from 1.98 in many chromium compounds to sometimes 6 in $\mathrm{Co}^{++}$depending upon the nature of the spin system used. A theoretical study of the constants is rather difficult because exact information about the nuclear magnetic dipole and electric quadrupole moments, spin-spin and spin-orbit interactions is not available.

Recently Narsimhamurty and Premswarup ${ }^{1}$ developed a general method for calculating the constants of the Spin-Hamiltonian. The method applies the first order perturbation theory involving the relative positions of certain energy levels, and so it is approximate and the general method is applied to three compounds of copper ion viz. copper tetramine sulphate monohydrate, copper ammonium tutton salt, and copper diethylenediamine chloride doubly hydrated.

\section{Theoretical Aspect}

The principal values of the $g$ tensor are related to energy values of the levels in which the configuration ground state is split under a tetragonal symmetric crystalline electric field. Approximately

$$
\begin{aligned}
& g_{\|}=2-8 \lambda /\left(E_{2}-E_{0}\right), \\
& g_{\perp}=2-2 \lambda /\left(E_{3}-E_{0}\right),
\end{aligned}
$$

where $g_{\|}$and $g_{\perp}$ are $g$ values parallel and perpendicular to the tetragonal axis of symmetry. $E_{0}$ is the energy of the lowest orbital level and $E_{2}$ and $E_{3}$ are those of higher levels split from the upper triplet. The spin-orbit coupling constant $\lambda$ is equal to $-829 \mathrm{~cm}^{-1}$ for the free copper ion, but varies with the lattice. The Hamiltonian of a paramagnetic ion in a lattice palced in an external magnetic field can be written as

$$
H=H_{\text {elect }}+H_{\text {cf }}+H_{\mathrm{LS}}+H_{\mathrm{SS}}+\beta H(L+2 S)
$$

where the symbols have their usual significance. As paramagnetic resonance studies are mainly concerned with the lowest level and the levels near it, one is required to consider the levels which are produced by the $H_{\text {elect }}+H_{\text {cf }}$ part of the Hamiltonian, the remaining smaller terms being used as perturbation over the splitting produced by them. The problem is further simplified by relating the matrix elements of $H_{\mathrm{cf}}$ in the secular equation to the eigen-states of $H_{\text {elect }}$ which is degenerate in our case. The matrix elements of the reduced Hamiltonian are formed from the crystalline field parameters which are related to the field for tetragonal symmetry as

$V_{\text {cryst }}=A_{2}{ }^{0} \overline{r^{2}} Y_{2}{ }^{0}+A_{4}{ }^{0} \overline{r^{4}} Y_{4}{ }^{0}+A_{+}^{4}{ }^{4} r^{4} Y_{4}^{ \pm 4}$

where $A_{2} \overline{0} r^{2}, A_{4}{ }^{0} r^{4}$ and $\overline{A_{4}} \pm 4 r^{4}$ are crystalline field meters. These can be calculated for atoms and molecules as

$$
\begin{aligned}
& A_{2}{ }^{0} \overline{r^{2}}=6(\pi / 5)^{\frac{1}{2}}\left[\frac{2 \mu_{\mathrm{A}}}{a^{4}}-\frac{\mu_{\mathrm{B}}}{b^{4}}-\frac{\mu_{\mathrm{C}}}{c^{4}}\right] \overline{r^{2}}, \\
& A_{4} \overline{{ }^{0} r^{4}}=-\frac{10}{3} V \pi\left[\frac{3}{2} \frac{\mu_{\mathrm{A}}}{a^{6}}+\frac{\mu_{\mathrm{B}}}{b^{6}}+\frac{\mu_{\mathrm{C}}}{c^{6}}\right] \overline{r^{4}}, \\
& A_{4} \pm 4 \overline{r^{4}}=\frac{5}{3}\left(\frac{35 \pi}{2}\right)^{\overline{\frac{1}{2}}} \frac{r^{4} \mu_{\mathrm{A}}}{a^{6}}
\end{aligned}
$$

where $\overline{r^{2}}$ and $\overline{r^{4}}$ are the average values of $r^{2}$ and $r^{4}$ for $3 d$ eletrons and are equal to $0.9 \times 10^{-16} \mathrm{~cm}^{2}$ and $1.3 \times 10^{-32} \mathrm{~cm}^{4}$ respectively ${ }^{2}$, and $\mu_{\mathrm{A}}, \mu_{\mathrm{B}}, \mu_{\mathrm{C}}$ are the 
effective dipole moments of different atoms or molecules as indicated in Figure 1. The general expression for calculating the effective dipole moments can be written as

$$
\mu_{t}^{\mathrm{eff}}=\mu_{t}^{\mathrm{perm}}+p_{t} \sum_{s=t} E_{s t}
$$

where $p_{t}$ is the polarizability of the $t$-th particle and $E_{s t}$ represents the field at the $t$-th particle due to the charge $q_{s}$ and dipole moment $\mu_{s}$ of the $s$-th particle.

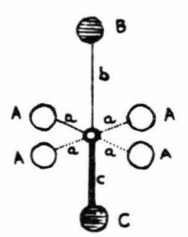

Fig. 1. Atomic configuration surrounding a Copper ion in general for the three cases considered. $\mathrm{Cu}(\mathrm{en})_{2} \mathrm{Cl}_{2} \cdot 2 \mathrm{H}_{2} \mathrm{O}: \mathrm{A}$ $=\mathrm{N}, \mathrm{B}=\mathrm{Cl}, \mathrm{C}=\mathrm{H}_{2} \mathrm{O}, \mathrm{Cu}\left(\mathrm{NH}_{3}\right)_{4} \mathrm{SO}_{4} \cdot \mathrm{H}_{2} \mathrm{O}: \mathrm{A}=\mathrm{N}, \mathrm{B}=\mathrm{H}_{2} \mathrm{O}$, $\mathrm{C}=\mathrm{H}_{2} \mathrm{O}, \mathrm{Cu}\left(\mathrm{NH}_{4}\right)_{2}\left(\mathrm{SO}_{4}\right)_{2} \cdot 6 \mathrm{H}_{2} \mathrm{O}: \mathrm{A}=\mathrm{H}_{2} \mathrm{O}, \mathrm{B}=\mathrm{H}_{2} \mathrm{O}, \mathrm{C}=$ $\mathrm{H}_{2} \mathrm{O}$.

The configurational ground state of $\mathrm{Cu}^{++}$is ${ }^{2} \mathrm{D}$. Since the ion has 9 electrons in its outermost orbit it can be treated as a positive hole in a complete shell and can be represented by a single electron wave function. A $5 \times 5$ matrix is then calculated from the equation

$$
\begin{array}{r}
V_{m^{\prime} m^{\prime \prime}}=A_{2}{ }^{0} \overline{r^{2}} \iint Y_{2}{ }^{m^{\prime *}} Y_{2}{ }^{0} Y_{2}{ }^{m^{\prime \prime}} \operatorname{Sin} \Theta \mathrm{d} \Theta \mathrm{d} \varphi \\
+A_{4}{ }^{0} \overline{r^{4}} \iint Y_{2}{ }^{m^{\prime *}} Y_{4}{ }^{0} Y_{2}{ }^{m^{\prime \prime}} \operatorname{Sin} \Theta \mathrm{d} \Theta \mathrm{d} \varphi \\
+A_{4}{ }^{ \pm 4} r^{4} \overline{\iint Y_{2}{ }^{m^{\prime *}} Y_{4}{ }^{ \pm 4} Y_{2}{ }^{m^{\prime \prime}}} \operatorname{Sin} \Theta \mathrm{d} \Theta \mathrm{d} \varphi
\end{array}
$$

with the help of Table $3^{1}$ and the orthonormal properties of the splerical harmonics. The matrix on solution yields four different eigenvalues, one of which corresponds to a doublet and the other three to singlets. Susceptibility can also be calculated from the energy values as calculated with the help of the following equations ${ }^{3}$

$$
\begin{gathered}
\chi \|=\left(1-\frac{4 \lambda}{E_{2}-E_{0}}\right)^{2} \frac{0.376}{T}+\frac{2.1}{E_{2}-E_{0}} \\
\chi \perp=\left(1-\frac{\lambda}{E_{3}-E_{0}}\right)^{2} \frac{0.376}{T}+\frac{0.53}{E_{3}-E_{0}}
\end{gathered}
$$

assumed to be true for all the three cases.

\section{Results and Discussion}

i) In a molecule of copper diethylene diamine chloride ${ }^{4}$, the $\mathrm{Cu}$ atom is surrounded by 4 nitrogen atoms of the two ethylenediamine groups which make a square coordination. On one side of this plane there is one chlorine at a distance of $2.89 \AA$ from the copper atom and on the other side a water molecule at a distance of $2.68 \AA$. $N$ atoms are at a distance of $1.99 \AA$ from the $\mathrm{Cu}$ atom. From the molecular shape a tetragonal symmetric field can be assumed to be acting on $\mathrm{Cu}^{+}$. The basic concept behind the point charge point dipole model is that $d$ wave functions don't overlap with the wave functions of the surrounding ligands, which is not strictly the case. Hence the wave functions of the whole complex must be considered. This is studied as the effect of covalent bonding by OwEN 5 and many others. Owen has suggested to replace $\lambda$ by $\alpha^{2} \lambda^{\prime}$ where $\alpha^{2}$ is called covalency factor and accounts for the covalent bonding. $\lambda^{\prime}$ is nearly the same as $\lambda$. In this case ${ }^{6} \alpha^{2} \lambda^{\prime}=-414 \mathrm{~cm}^{-1} \alpha^{2}$ is calculated by substituting the experimental values of $g$ and $\Delta E$ in Eq. (1) or (2) and dividing the values of $\lambda$ so calculated by the free ion value of $\lambda$. The expressions after accounting for covalent effects

$$
\begin{aligned}
& g_{\|}=2-8 \alpha^{2} \lambda^{\prime} /\left(E_{2}-E_{0}\right), \\
& g_{\perp}=2-2 \alpha^{2} \lambda^{\prime} /\left(E_{3}-E_{0}\right),
\end{aligned}
$$

have been used. The results calculated are given in Tables 1, 2 and 3. The required polarizabilities and permanent dipole moments are

$$
\begin{aligned}
P_{\mathrm{N}} & =1.76 \times 10^{-24} \text { c.c. }, \quad \mu_{\mathrm{N}}=0, \\
P_{\mathrm{Cl}} & =3.01 \times 10^{-24} \text { c.c. }, \quad \mu_{\mathrm{Cl}}=0, \\
P_{\mathrm{H} 2 \mathrm{O}} & =1.48 \times 10^{-24} \text { c.c. }, \quad \mu_{\mathrm{H} 2 \mathrm{O}}=1.87 \mathrm{D} .
\end{aligned}
$$

ii) A unit cell of $\mathrm{Cu}\left(\mathrm{NH}_{3}\right)_{4} \mathrm{SO}_{4} \cdot \mathrm{H}_{2} \mathrm{O}$ contains 4 formla molecules ${ }^{4}$. There is a tetragonal symmetric crystalline field acting on the $\mathrm{Cu}$ ion. There are four coplanar nitrogen atoms of amine group at a distance of $2.05 \AA$ from the copper atom. One $\mathrm{H}_{2} \mathrm{O}$ is above this plane and the other below it at a distance of 2.59 $\AA$ and $3.37 \AA$ respectively on the tetragonal axis. $\alpha^{2} \lambda^{\prime}$ $=-390 \mathrm{~cm}^{-1}$ is necessary in this case to explain both the values of $g_{\|}$and $g_{\perp}$ observed experimentally. The data calculated appear in Tables 1, 2 and 3.

iii) In case of $\mathrm{Cu}\left(\mathrm{NH}_{4}\right)_{2}\left(\mathrm{SO}_{4}\right)_{2} \cdot 6 \mathrm{H}_{2} \mathrm{O}$, the crystalline field acting at the copper is ion more or less cubic. There are 6 water molecules all at equal distances of nearly $2 \AA$ and forming a distorted octahedron. The cubic field leaves a nonmagnetic orbital doublet lowest. This gives rise to a Jahn-Teller effect, due to which 
Table 1. The effective dipole moments $(\mu)$ and crystalline field parameters (Ar) for the three complexes.

\begin{tabular}{|c|c|c|c|c|c|c|}
\hline \multirow{2}{*}{$\begin{array}{l}\text { Effective dipole moments } \\
\text { and cryst. field parameters } \\
\mu_{\mathrm{N}}\end{array}$} & \multicolumn{2}{|c|}{$\mathrm{Cu}(\mathrm{en})_{2} \mathrm{Cl}_{2} \cdot 2 \mathrm{H}_{2} \mathrm{O}$} & \multicolumn{2}{|c|}{$\mathrm{Cu}\left(\mathrm{NH}_{3}\right)_{4} \mathrm{SO}_{4} \cdot \mathrm{H}_{2} \mathrm{O}$} & \multicolumn{2}{|c|}{$\mathrm{Cu}\left(\mathrm{NH}_{4}\right)_{2}\left(\mathrm{SO}_{4}\right)_{2} \cdot 6 \mathrm{H}_{2} \mathrm{O}$} \\
\hline & 2.74 & $\mathrm{D}$ & 2.91 & $\mathrm{D}$ & & \\
\hline$\mu \mathrm{H}_{2} \mathrm{O}$ & 2.95 & $\mathrm{D}$ & $\ldots$ & & 3.77 & $\mathrm{D}$ \\
\hline$\mu_{\mathrm{Cl}}$ & 2.28 & $\mathrm{D}$ & & & $\ldots$ & \\
\hline$\mu_{\mathrm{H}_{2} \mathrm{O}}$ & $\ldots$ & & 3.25 & $\mathrm{D}$ & $\ldots$ & \\
\hline$\mu_{\mathrm{H}_{2} \mathrm{O}}$ & $\ldots$ & & 2.70 & D & $\ldots$ & \\
\hline$A_{2}{ }^{0} \overline{r^{2}}$ & 26904 & $\mathrm{~cm}^{-1}$ & 23000 & $\mathrm{~cm}^{-1}$ & $\ldots$ & \\
\hline$A_{4}{ }^{0} \overline{r^{4}}$ & -14511 & $\mathrm{~cm}^{-1}$ & -13200 & $\mathrm{~cm}^{-1}$ & -38266 & $\mathrm{~cm}^{-1}$ \\
\hline$A_{4} \pm 4 \overline{r^{4}}$ & 17159 & $\mathrm{~cm}^{-1}$ & 16000 & $\mathrm{~cm}^{-1}$ & 22871 & $\mathrm{~cm}^{-1}$ \\
\hline
\end{tabular}

Table 2. The Eigenvalues for the three complexes.

\begin{tabular}{cccc}
\hline Level & $\mathrm{Cu}(\mathrm{en})_{2} \mathrm{Cl}_{2} \cdot 2 \mathrm{H}_{2} \mathrm{O} \mathrm{cm}{ }^{-1}$ & $\mathrm{Cu}\left(\mathrm{NH}_{3}\right)_{4} \mathrm{SO}_{4} \cdot \mathrm{H}_{2} \mathrm{O} \mathrm{cm}-1$ & $\mathrm{Cu}\left(\mathrm{NH}_{4}\right)_{2}\left(\mathrm{SO}_{4}\right)_{2} \cdot 6 \mathrm{H}_{2} \mathrm{O} \mathrm{cm}^{-1}$ \\
\hline$E_{3}$ & 4815 & 4150 & 6790 \\
$E_{2}$ & 1338 & 850 & 3689 \\
$E_{1}$ & 252 & 846 & -533 \\
$E_{0}$ & -11634 & -11096 & -7829 \\
\hline
\end{tabular}

Table 3.The calculated values of $g, \Delta E$ and $\chi$ and experimental values of $g, \Delta E$ and $\alpha^{2}$.

\begin{tabular}{|c|c|c|c|c|c|c|c|c|c|}
\hline \multirow{3}{*}{$\begin{array}{l}\text { Complex } \\
\mathrm{Cu}(\mathrm{en})_{2} \mathrm{Cl}_{2} \cdot 2 \mathrm{H}_{2} \mathrm{O}\end{array}$} & \multirow{3}{*}{$\begin{array}{c}\alpha^{2} \\
0.5\end{array}$} & \multirow{3}{*}{$\begin{array}{r}\Delta E_{\text {exp }} \\
14786\end{array}$} & \multirow{3}{*}{$\begin{array}{c}\begin{array}{c}\Delta E_{\mathrm{cal}} \\
\mathrm{cm}^{-1}\end{array} \\
14710\end{array}$} & \multicolumn{2}{|c|}{ Calculated } & \multicolumn{2}{|c|}{ Experimental } & \multirow{2}{*}{\multicolumn{2}{|c|}{ 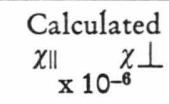 }} \\
\hline & & & & $g_{\|}$ & $g \perp$ & $g_{\|}$ & $g \perp$ & & \\
\hline & & & & 2.26 & 2.05 & 2.25 & 2.05 & 1756 & 1348 \\
\hline $\mathrm{Cu}\left(\mathrm{NH}_{3}\right)_{4} \mathrm{SO}_{4} \cdot \mathrm{H}_{2} \mathrm{O}$ & $0.5^{10}$ & 14200 & 13946 & 2.23 & 2.05 & 2.22 & 2.05 & 1770 & 1351 \\
\hline $\mathrm{Cu}\left(\mathrm{NH}_{4}\right)_{2}\left(\mathrm{SO}_{4}\right)_{2} \cdot 6 \mathrm{H}_{2} \mathrm{O}$ & $0.8^{9}$ & 12300 & 12600 & 2.45 & 2.09 & 2.45 & 2.09 & 2160 & 1412 \\
\hline
\end{tabular}

the positions of the water molecules are shifted to lower symmetry positions (Tetragonal, where 4 water molecules are shifted at distances of $1.9 \AA$ and two others at a distance of $2.15 \AA$ from $\mathrm{Cu}^{++}$) which leads to the removal of orbital degeneracy. The estimation of the shift in energy due to the Jahn-Teller effect is given by VanVleck ${ }^{7}$

$$
D q=D^{\prime} \overline{r^{4}}(2 / 105)=1500 \mathrm{~cm}^{-1} \text { in our case }
$$

where $D^{\prime}$ and $\overline{r^{4}}$ have their usual meaning. $\alpha^{2} \lambda^{\prime}$ in this case has been calculated to be $-695 \mathrm{~cm}^{-1}$. Accounting for this effect the data calculated are given in Tables 1,2 and 3.

Comparison of the results indicate that the calculated values agree fairly well with the experimental values of $g$ and the discrepancy is within the range of experimental error. This is so because of the application of a covalency factor which describes the covalency in a particular molecule. $\left(1-\alpha^{2}\right)$ is a measure of the degree of mixing of the orbits or the strength of the bonds which are formed. $\alpha^{2}=1$ for a purely ionic complex and $\alpha^{2}=0.5$ for a purely covalent complex. The presence of covalent bonds with the neighbours of the paramagnetic ions also reduces the effective charges of the neighbours with the result that the energy gap of the splittings is reduced and a higher value of $g$ is obtained. It was argued ${ }^{8}$ that the reduction in $\lambda$ and the effective charges are in the same ratio and hence the covalent bonding does not affect the results but in the absence of any quantitative treatment, we believe that the former factor is more important than the latter and reduces the experimental values of $g$.

\section{Acknowledgements}

The authors SDS and SKG are thankful to the Department of Atomic Energy and C.S.I.R. for financial assistance.

1 A. Narsimhamurty and D. Premswarup, Indian J. Pure and Appl. Phys. 1, 100 [1963].

2 D. POLDER, Physica 9, 709 [1942].

3 B. Bleany, R. P. Penrose, and B. I. Plumpton, Proc. Roy. Soc. London 198, 406 [1949].

${ }^{4}$ H. ABE and K. ÔNo, J. Phys. Soc. Japan 11, 947 [1956].

5 J. Owen, Proc. Roy. Soc. London A227, 183 [1966].

6 K. Sone and S. Utsuno, Bull. Chem. Soc. Japan 39, 1813 [1966].

7 J. H. VANvleck, J. Chem. Phys. 7, 72 [1939].

8 L. S. Ramkrishna Rao and D. Premswarup, Indian J. Pure and Appl. Phys. 4, 296 [1966].

9 A. Abragam and M. H. L. Pryce, Proc. Roy. Soc. London 206, 164 [1951].

10 T. Dreisch and W. Trommer, Z. Phys. Chem. B37, 37 [1937]. 